\title{
Measuring the Glomerular Filtration Rate in Obese Individuals without Overt Kidney Disease
}

\author{
Allon N. Friedman ${ }^{a}$ Matthew Strother ${ }^{b}$ Sara K. Quinney ${ }^{b, c}$ Stephen Hall ${ }^{b, e}$ \\ Susan M. Perkins ${ }^{c}$ Edward J. Brizendine ${ }^{c}$ Margaret Inman ${ }^{g}$ Gerardo Gomez $^{d}$ \\ Zak Shihabi ${ }^{\text {h }}$ Sharon Moe ${ }^{a, f} \quad$ Lang Lic $^{c}$ \\ Divisions of a Nephrology, ${ }^{b}$ Pharmacology and ${ }^{\mathrm{C} B i o s t a t i s t i c s, ~ a n d ~ d ~ D e p a r t m e n t ~ o f ~ S u r g e r y, ~ I n d i a n a ~ U n i v e r s i t y ~}$ \\ School of Medicine, e Eli Lilly Co., and f Department of Medicine, Roudebush Veterans Affairs Medical Center, \\ Indianapolis, Ind., 9St. Vincent Carmel Bariatric Center of Excellence, St. Vincent Hospital, Carmel, Ind., and \\ hDepartment of Pathology, Wake Forest School of Medicine, Winston-Salem, N.C., USA
}

\section{Key Words}

lohexol $\cdot$ Glomerular filtration rate $\cdot$ Creatinine $\cdot$ Cystatin C . Obesity

\begin{abstract}
Background: Identifying methods to accurately measure the glomerular filtration rate (GFR) in obese individuals without kidney overt kidney disease is necessary to understanding the pathophysiology and natural history of obesity-related kidney disease. Methods: Using a cross-sectional design, iohexol clearance and disposition was measured, an optimal sampling schedule was identified, and the reliability of GFRestimating methods was described in 29 obese individuals with normal serum creatinine levels. Iohexol disposition was measured using population pharmacokinetics. The agreement with GFR-estimating equations was assessed by intraclass coefficients. Results: Mean age was $44 \pm 10$ years, body mass index $45 \pm 10$, creatinine $0.7 \pm 0.2 \mathrm{mg} / \mathrm{dl}(62 \pm$ $18 \mu \mathrm{mol} / \mathrm{l})$, and cystatin C $0.83 \pm 0.18 \mathrm{mg} / \mathrm{dl}(8.3 \pm 1.8 \mathrm{mg} / \mathrm{l})$. lohexol disposition fit a two-compartment model and 5 sampling windows were identified over a 4-hour period to
\end{abstract}

optimize model accuracy and minimize blood draws. Precision was not compromised with this sampling design. Neither creatinine nor cystatin $C$ were linearly correlated with the measured GFR though cystatin C was independent of body composition. Agreement was fair to poor between the measured GFR and GFR-estimating equations. Conclusion: This study offers a rigorous method to study obesity-related kidney disease and improve upon suboptimal GFR-estimating methods.

Copyright $\odot 2010$ S. Karger AG, Basel

\section{Introduction}

The obesity public health crisis, which is expected to encompass 700 million adults by the year 2015 [1], has profound global implications for kidney disease. Aside from being a major risk factor for the development of diabetes and hypertension, the principal causes of chronic kidney disease (CKD) in the Western world, obesity may have adverse effects on kidney function independent of such intermediate disease states $[2,3]$.

\section{KARGER}

๑ 2010 S. Karger AG, Basel

Fax +41613061234

E-Mail karger@karger.ch

www.karger.com 
Unfortunately, fundamental knowledge about obesity-related kidney disease, including its prevalence, natural history, risk factors, and effective preventative strategies, is sorely lacking. Insights into these issues will require the ability to accurately measure and estimate the glomerular filtration rate (GFR), the best global indicator of kidney function [4], in obese individuals with incipient kidney disease or even normal kidney function. Iohexol, a nonionic low-osmolar contrast agent, is an ideal filtration marker for this task in that it is readily obtainable, inexpensive, nonradioactive, and does not require cumbersome urine collections. It has been used for years as a reliable GFR marker in children and adults, particularly in Europe [5-7].

There is ample reason to believe that the current GFRestimating methods, derived primarily in lean individuals with CKD, may be less accurate in extreme states such as obesity [8]. Even reference clearance markers such as iohexol have never been formally tested in the obese population. This is a concern because the volume of distribution and/or tissue handling of clearance markers may be affected by obesity, leading to altered pharmacokinetics and renal clearance.

The project goal was to study plasma iohexol as a GFR marker in obese humans with apparently normal kidney function. In doing so, it would help establish (1) a model of iohexol disposition and an optimal iohexol sampling schedule in obese subjects that would minimize the number of blood draws yet maximize overall accuracy, and (2) the reliability of commonly used GFR-estimating methods in obesity.

\section{Subjects and Methods}

\section{Study Population}

Twenty-nine obese individuals were recruited from the Indianapolis, Ind., area between April 2004 and October 2007 as part of a larger National Institutes of Health-sponsored study (NCT00244790). The relevant institutional review boards approved the protocol and all patients gave written informed consent after reviewing a written summary of the plan. Exclusion criteria included the following: age less than 18 years, pregnant state, body mass index (BMI) less than 30 , inability to give informed consent, iodine or contrast allergy, serum creatinine $>1.3$ $\mathrm{mg} / \mathrm{dl}(115 \mu \mathrm{mol} / \mathrm{l})$ in women and $>1.5 \mathrm{mg} / \mathrm{dl}(133 \mu \mathrm{mol} / \mathrm{l})$ in men. Each subject had up to 6 iohexol-based GFR measurements performed over a range of weeks to months.

\section{Study Protocol}

All subjects presented after an overnight fast to the Indiana University General Clinical Research Center. Subjects were encouraged to hydrate themselves prior and during the visit to avoid dehydration. Vital signs were measured and 2 peripheral intravenous lines were inserted, one for the iohexol bolus injection and one for the blood sampling. Approximately 5,000 mg iohexol (Omnipaque-300; GE Healthcare, Piscataway, N.J., USA) was injected slowly over $2 \mathrm{~min}$ from a preweighed syringe at time 0 , followed by a $20-\mathrm{ml}$ normal saline flush. The syringe was then weighed to the nearest ten-thousandths gram on the same scale used prior to the injection. The exact dose of iohexol (in grams) was calculated from the difference in syringe weights multiplied by the amount of iohexol in Omnipaque-300 (647 mg/g Omnipaque) divided by its density at room temperature (1.349). A total of 90 iohexol clearance measurements were performed in a variety of study protocols in the 29 study subjects, each on separate days. As the project evolved, the number of iohexol samplings per clearance measurement increased due to the understanding that this would increase the overall accuracy. The first 25 clearance measurements were based on 2 blood samples drawn at 120 and 240 min (subjects 1-8), the next 54 on 10 samples drawn at $15,30,45$, $60,90,120,150,180,210$, and $240 \mathrm{~min}$ (subjects 8-26), and the remaining 11 on 11 samples (the previous protocol plus an extra sample drawn at $5 \mathrm{~min}$ ) (subjects 25, 27-29). Serum was isolated and aliquots were stored at $-80^{\circ} \mathrm{C}$.

BMI and body surface area (BSA) were calculated [9] during each visit from measured weight and height while wearing a hospital gown and no shoes. Body composition was measured using bioelectrical impedance analysis (BIA-101Q Fluid/ Nutrition BioElectrical Impedance Analyzer System with Cyprus 1.0 Body Composition Analysis Software, RJL Systems, Clinton Township, Mich., USA), which has been studied in the obese population [10]. Diabetes was defined as a previous diagnosis of diabetes or a fasting plasma glucose $\geq 126 \mathrm{mg} / \mathrm{dl}$ (7 $\mathrm{mmol} / \mathrm{l})$ [11]. Hypertension was defined as a previous diagnosis of hypertension or 2 or more blood pressure readings of $\geq 140 / 90 \mathrm{~mm} \mathrm{Hg}$ [12] on at least one visit.

\section{Laboratory Measurements}

As previously described [13], iohexol in serum was measured by capillary electrophoresis using a Model $2050 \mathrm{CE}$ instrument (Beckman Instruments, Palo Alto, Calif., USA) by injecting the serum directly on untreated capillary followed by separation based on micellar electrokinetic chromatography. In this technique, iohexol in the serum is distributed between the separation buffer in the capillary and the sodium dodecyl sulfate micelle according to its distribution coefficient. It migrates based on the velocity of the micelle under the influence of the electric field. After separation from the other compounds present in the serum, iohexol is detected on the capillary by its absorption at $254 \mathrm{~nm}$. The method is linear between 8 and $260 \mathrm{mg} / \mathrm{l}$, with a relative standard deviation of peak height of $2.9 \%$.

Cystatin $\mathrm{C}$ was measured using particle-enhanced immunonephelometry (Siemens/Dade Behring BNII Nephelometer, Ill., USA), and serum creatinine by the Roche/Hitachi Creatinine Plus enzymatic assay (Roche Diagnostics, Basel, Switzerland), the results of which correlate with those obtained by isotopedilution mass spectrometry. Urine albumin and creatinine were measured using standard laboratory techniques. The GFR was predicted using the modification of diet in renal disease (MDRD) equation modified for standardized serum creatinine measurements [14], while the creatinine clearance was estimated using 
the Cockcroft-Gault method [15]. Given the concern that indexing GFR by BSA may introduce bias when used in obese persons [16], we used nonindexed measurements and estimations as a rule. We removed indexing from the MDRD equation by multiplying it by BSA/1.73.

\section{Population Pharmacokinetic Modeling and Statistics}

Iohexol clearance was determined by a nonlinear mixed-effects modeling approach for population pharmacokinetic analysis using NONMEM VI (Globomax LLC, Ellicott City, Md., USA) $[22,23]$. A two-compartment base model (ADVAN4 TRANS4) with a first-order conditional estimation method was found to best describe the iohexol plasma-concentration time curves. Iohexol dose, dosing time, time of blood draws, and concentrations were entered into the model for each subject, and the individual pharmacokinetic parameters, including clearance (i.e. GFR) were estimated. This approach also determines interindividual and intraindividual variability of the pharmacokinetic parameters. Additional models were evaluated based on the initial two-compartment model to include subject-specific covariates, including age, weight, lean mass and fat mass. In addition, the following categorical covariates were explored: sex, hypertension, diabetes, and the impact of interoccasion variation. Model evaluation was performed utilizing both minimization of the objective function (OBJ; -2 log likelihood) and diagnostic plots. Individual covariates were determined to be significant if they caused a decline of the OBJ by at least $3.84\left(\mathrm{p}<0.05,1\right.$ d.f. in $\left.\chi^{2}\right)$. Continuous covariates were centered on their median value. A full model was then created with inclusion of all significant covariates. Selection between covariates that described the same biologically relevant concept (weight, lean body mass and fat body mass) was made based on that covariate which had the greatest decline in OBJ. Full model components were subsequently evaluated through stepwise backward elimination procedure, with covariates maintained in the final model which caused a change in OBJ of at least 10.83 ( $\mathrm{p}<0.001,1$ d.f.). Visual analysis was performed in $\mathrm{R}$ [17] using the Xpose4 package [18]. Individual predictions of iohexol clearance (GFR) were generated using the IPRED output from NONMEM. Determination of optimal sampling points for the GFR measurements using iohexol clearance was made with WinPOPT [19] using the simulated annealing algorithm in 1,000 simulated subjects utilizing parameter estimates from the final model produced by NONMEM. In an effort to determine an optimal blood sampling schedule within a clinically feasible study period, the latest sampling point was set to $4 \mathrm{~h}$. Sampling time windows were determined using the same sample window function of WinPOPT.

Agreement of the iohexol GFR (estimated from study subjects through the prescribed population pharmacokinetic model) with the MDRD GFR and Cockcroft-Gault creatinine clearance was assessed by estimating the intraclass coefficient (ICC) and its 95\% confidence interval (CI). ICCs were calculated on the statistical software program $\mathrm{R}$ version 2.8.0 [17] using the 'irr' package [20]. Additionally Bland-Altman plots [21] were constructed along with the Bland-Altman limits of agreement. Pearson's correlation coefficient was estimated to assess the strength of the linear relationship between GFR and physical characteristics. A significance level of 0.05 was used in all statistical tests.
Table 1. Baseline characteristics

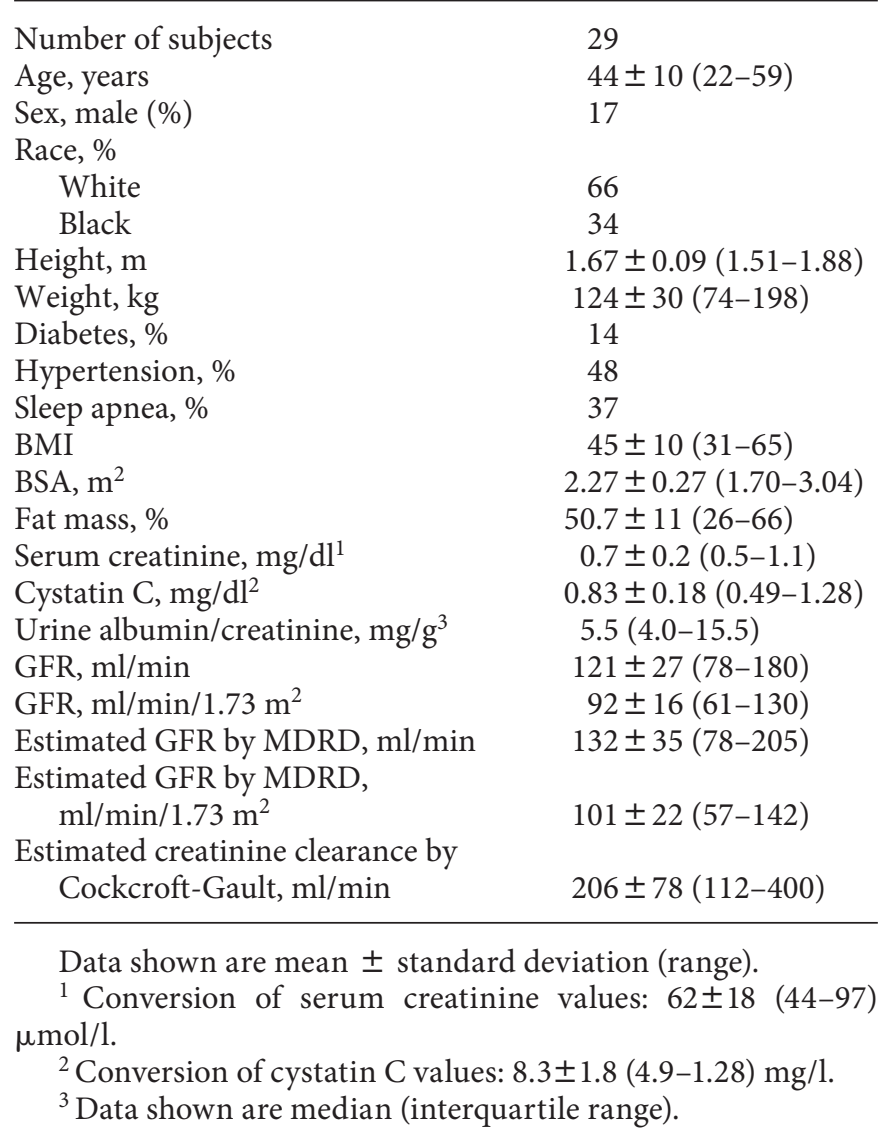

\section{Results}

\section{Patient Characteristics}

Table 1 describes at baseline the 29 study subjects, who were predominantly young-adult to middle-aged white females. The majority of subjects manifested the more severe degrees of obesity [World Health Organization classes II $(n=6)$ and III $(n=19)]$ [24]. Despite this, only a small minority had diabetes mellitus, while a larger proportion had mild hypertension and sleep apnea. Serum creatinine and cystatin $C$ levels were well within the normal range. Albuminuria was below the microalbuminuria range for most subjects (19 with $\leq 10$ $\mathrm{mg} / \mathrm{g}, 7$ between 10 and $30 \mathrm{mg} / \mathrm{g}$, and $3 \geq 30 \mathrm{mg} / \mathrm{g})$. Of the 10 subjects with albuminuria of $10 \mathrm{mg} / \mathrm{g}$ or greater, only 2 had diabetes and/or hypertension. Seven individuals had GFR levels between 78 and $94 \mathrm{ml} / \mathrm{min}, 7$ between 105 and $113 \mathrm{ml} / \mathrm{min}$, and 15 subjects between 120 and $180 \mathrm{ml} / \mathrm{min}$, suggesting that glomerular hyperfiltration was fairly common. Excluding subjects with 
Table 2. Population model estimates for iohexol-based clearance

\begin{tabular}{llc}
\hline Equation variable & Parameter & Parameter value \\
\hline$\theta_{1}$ & $\mathrm{CL}, \mathrm{l} / \mathrm{h}$ & $7.06 \pm 0.678$ \\
$\theta_{2}$ & $\mathrm{~V}_{1}, \mathrm{l}$ & $9.87 \pm 3.12$ \\
$\theta_{3}$ & $\mathrm{Q}, \mathrm{l} / \mathrm{h}$ & $11.4 \pm 3.1$ \\
$\theta_{4}$ & $\mathrm{~V}_{2}, \mathrm{l}$ & $6.15 \pm 0.876$ \\
$\theta_{1} \exp ^{(\theta 5 \cdot \mathrm{WT})}$ & Influence of weight $(\mathrm{WT})$ on clearance & $0.00259 \pm 0.00254$ \\
$\Theta_{2} \cdot\left(1+\theta_{6} \cdot \mathrm{LBM}\right)$ & Influence of lean body mass $(\mathrm{LBM})$ on $\mathrm{V}_{1}$ & $0.0249 \pm 0.01634$ \\
\hline
\end{tabular}

Values are mean \pm standard deviation. $\mathrm{CL}=$ Drug clearance (for iohexol, renal clearance assumed); $\mathrm{V}_{1}=$ volume of the central compartment; $\mathrm{Q}=$ flow between peripheral and central compartment; $\mathrm{V}_{2}=$ volume of the peripheral compartment.

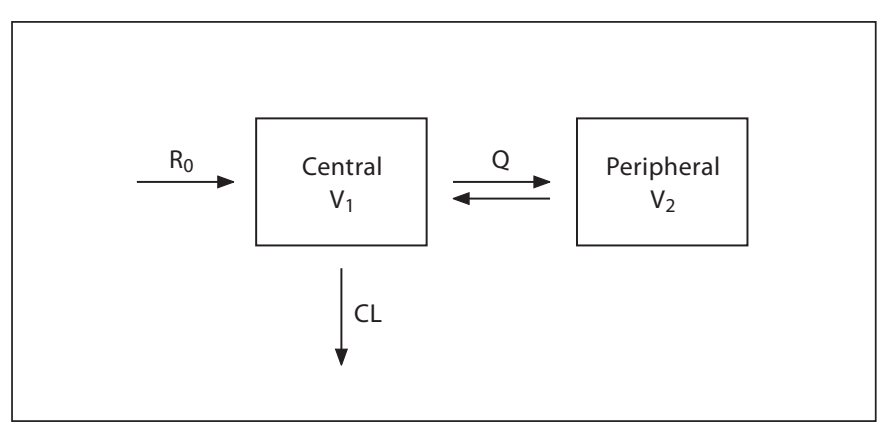

Fig. 1. Two-compartment i.v. infusion model. $\mathrm{R}_{0}=$ Rate of drug infusion; $\mathrm{V}_{1}=$ volume of the central compartment; $\mathrm{CL}=\mathrm{drug}$ clearance (for iohexol, renal clearance assumed); $\mathrm{Q}=$ flow between peripheral and central compartment; $V_{2}=$ volume of the peripheral compartment.

GFR measurements based on only 2 iohexol clearance time-point measurements did not appreciably affect these findings.

\section{Population Pharmacokinetic Modeling and Optimal \\ Sampling Schedule}

The optimal structural pharmacokinetic model based on bolus administration of iohexol was determined by visual fit to be a two-compartment model. Parameters of the two-compartment model were clearance, volume compartment 1 , volume compartment 2 , and intercompartmental clearance (Q) (fig. 1). Weight and lean body mass were found to be significant positive correlates of renal clearance and volume of distribution, respectively. The final parameter estimates for the population model are presented in table 2 (for standard errors and between subject variance, see additional data to table 2 ). Within-
Additional data to table 2

\begin{tabular}{|c|c|c|c|c|}
\hline & Theta & Eta & Value & $\begin{array}{l}\text { Standard } \\
\text { error }\end{array}$ \\
\hline \multicolumn{5}{|c|}{ Pharmacokinetic parameter } \\
\hline CL & 1 & & 7.0600 & 0.33900 \\
\hline $\mathrm{V}_{1}$ & 2 & & 9.8700 & 1.56000 \\
\hline$Q$ & 3 & & 11.400 & 1.55000 \\
\hline $\mathrm{V}_{2}$ & 4 & & 6.1500 & 0.43800 \\
\hline Weight on clearance & 5 & & 0.0026 & 0.00127 \\
\hline Lean body mass on $V_{1}$ & 6 & & 0.0259 & 0.00817 \\
\hline \multicolumn{5}{|l|}{ Parameter relevance } \\
\hline CL & & 1 & 0.034 & 0.01070 \\
\hline Occasion & & 2 & 0.007 & 0.00195 \\
\hline Occasion & & 3 & 0.007 & 0.00195 \\
\hline Occasion & & 4 & 0.007 & 0.00195 \\
\hline Occasion & & 5 & 0.007 & 0.00195 \\
\hline Occasion & & 6 & 0.007 & 0.00195 \\
\hline Occasion & & 7 & 0.007 & 0.00195 \\
\hline $\mathrm{V}_{1}$ & & 8 & 0.007 & 0.00195 \\
\hline \multicolumn{5}{|l|}{ Sigma (residual error) } \\
\hline 0.0078 & & & - & 0.00104 \\
\hline
\end{tabular}

subject (interoccasion) variability was $8.8 \%$. The population model slightly underestimates iohexol concentrations at high levels. This bias was absent in the individual models, indicating an adequate fit for each individual concentration-time curve. Figure 2 shows iohexol concentrations over time as both observed by our laboratory as well as for the individual and population prediction models. Within the constraints of a clinically feasible study (i.e. over $4 \mathrm{~h}$ ), the optimal sampling time points and sampling windows are presented in table 3 . Because 
Fig. 2. Iohexol concentrations versus time as measured by our laboratory for all subjects (a) and predictions based on individual pharmacokinetic parameters and their weight and lean body mass (b).

Table 3. Optimal sampling time windows

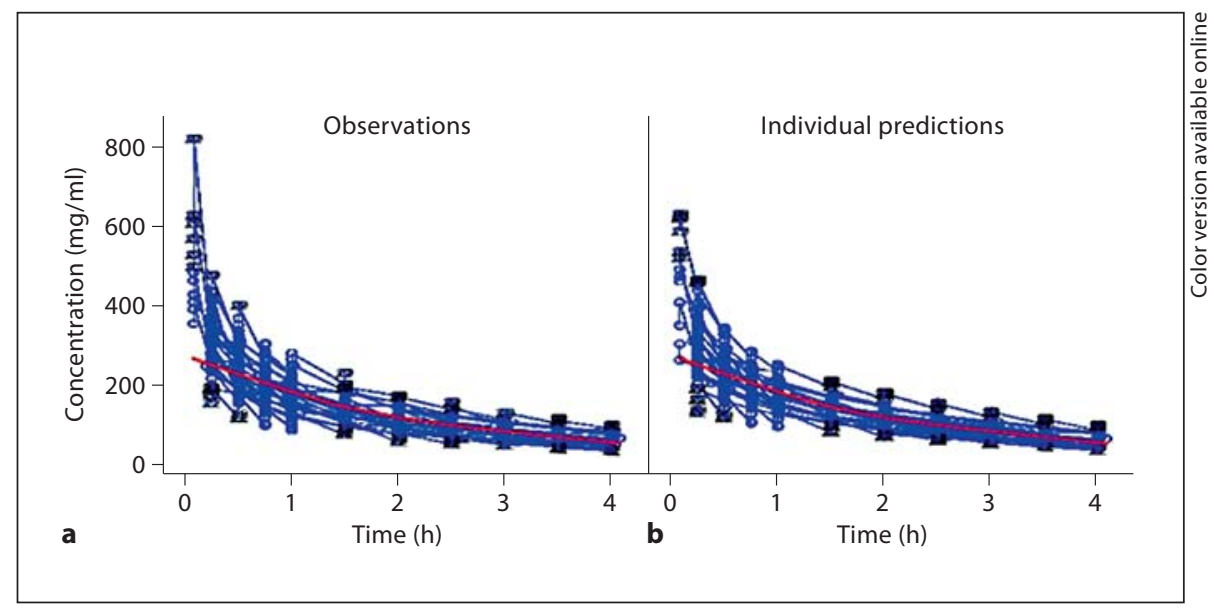

\begin{tabular}{lcc}
\hline Time point & Lower time limit & Upper time limit \\
\hline 1 & $15 \mathrm{~s}$ & $4 \mathrm{~min} 40 \mathrm{~s}$ \\
2 & $5 \mathrm{~min} 3 \mathrm{~s}$ & $17 \mathrm{~min} 52 \mathrm{~s}$ \\
3 & $45 \mathrm{~min} 43 \mathrm{~s}$ & $1 \mathrm{~h} 25 \mathrm{~min}$ \\
4 & $2 \mathrm{~h} 10 \mathrm{~min}$ & $3 \mathrm{~h} 4 \mathrm{~min}$ \\
5 & $4 \mathrm{~h} 7 \mathrm{~min}$ & $4 \mathrm{~h} 29 \mathrm{~min}$ \\
\hline
\end{tabular}

Windows are based on the assumption that time $0=$ the iohexol infusion. the two-compartment model contains 4 parameters, at least 5 sampling points are needed, the extra one being for residual variation in the model. In table 3, 2 closely spaced initial time points are necessary to obtain early on, followed by 3 subsequent measurements. A comparison of the precision of clearance based on a reference 10-point sampling design versus the optimal sampling design derived in this study is shown in table 4. As noted, precision in both sampling designs are quite similar, suggesting that precision was not compromised using the optimal design model. In addition, the BrochnerMortensen method (which presumes a one-compartment approach) is not as precise as our two-compartment approach (table 4).

\section{Reliability of Standard GFR-Estimating Methods in Obesity}

Comparisons of GFR by iohexol clearance, MDRD and Cockcroft-Gault equations are shown in figure 3. There was no significant linear correlation between the reference (i.e. iohexol) GFR and the inverse of either se- rum creatinine $(\mathrm{r}=0.08, \mathrm{p}=0.68)$ or cystatin $\mathrm{C}(\mathrm{r}=0.03$, $\mathrm{p}=0.88$ ) (fig. 4). Removing one obvious outlying data point strengthened the latter relationship, but not to statistical significance $(r=0.20, p=0.30)$. As noted in figure 5 , agreement of GFR with the Cockcroft-Gault-based estimated creatinine clearance was poor (ICC $=-0.13,95 \%$ CI: $-0.46,0.25)$ with a bias of $86 \mathrm{ml} / \mathrm{min}(\mathrm{p}<0.001,95 \%$ CI: $-49,220)$ and Bland-Altman limits of agreement of -49 to 220 . There was fair agreement between GFR and the MDRD GFR estimations (ICC $=0.51,95 \%$ CI: 0.19 , 0.74) (fig. 5), although there was a slight bias of $11.7 \mathrm{ml} /$ $\min (\mathrm{p}=0.041)$ and a wide spread in the limits of agreement $(-47.0,70.3)$. Furthermore, $72 \%$ (41\% when adjusting for BSA) and $24 \%$ of the GFR estimations by MDRD and Cockcroft-Gault, respectively, were within $30 \%$ of the true iohexol GFR. Adjusting for BSA did not improve agreement between the GFR and the 2 other measures.

In terms of physical characteristics, GFR was linearly associated with total weight $(\mathrm{r}=0.59, \mathrm{p}<0.001)$, lean body mass $(\mathrm{r}=0.65, \mathrm{p}<0.001)$, BMI $(\mathrm{r}=0.47, \mathrm{p}<0.001)$, and BSA $(r=0.60, p<0.001)$, with no association with 
Fig. 3. Comparison of GFR boxplot and individual data by iohexol clearance, MDRD and Cockcroft-Gault (C-G) equations.
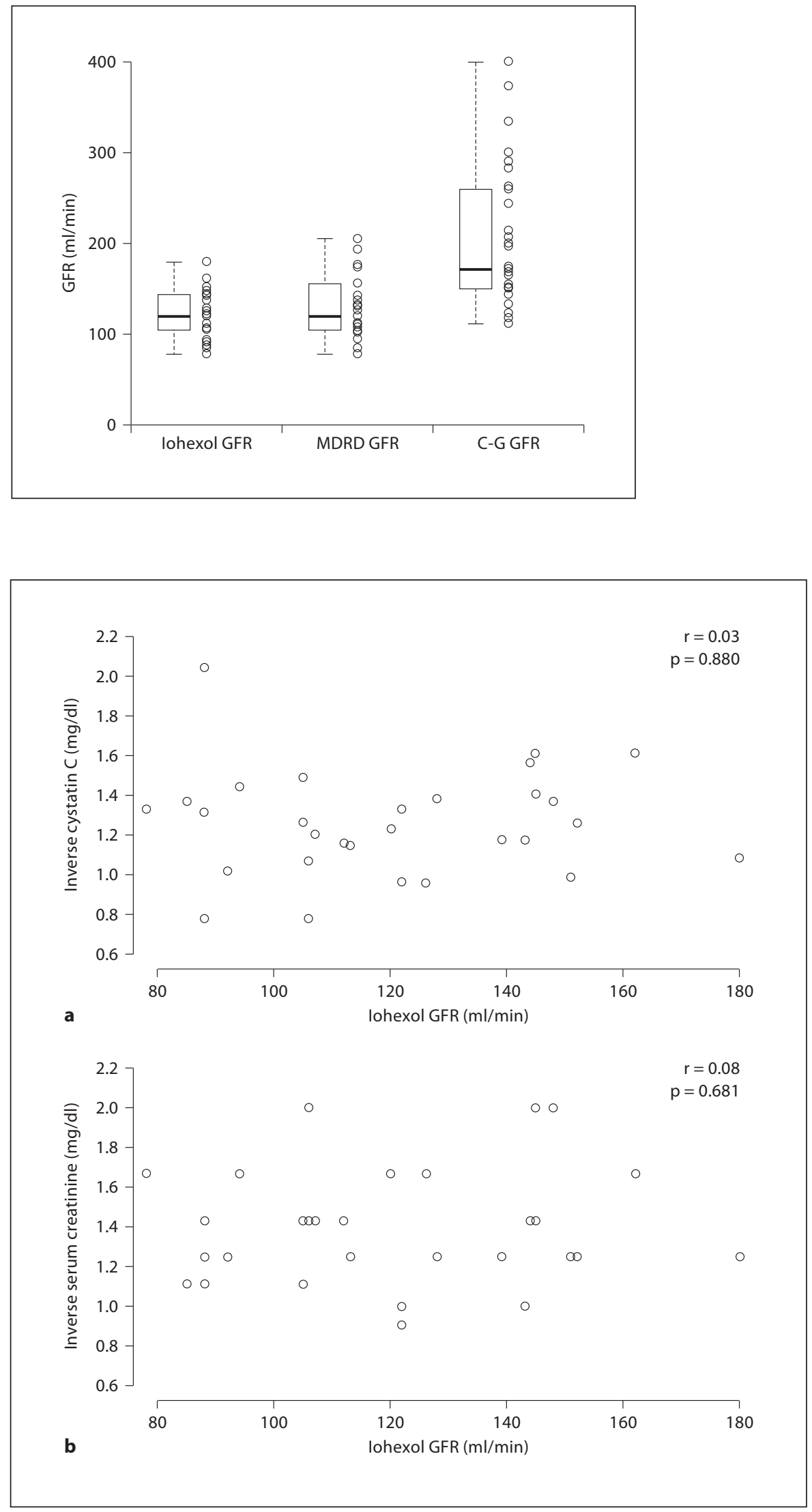

Fig. 4. a Relationship between inverse cystatin C and iohexol GFR. b Relationship between inverse serum creatinine and iohexol GFR. 
Fig. 5. a Bland-Altman plot of the difference between the MDRD-estimated GFR and the iohexol GFR. b Bland-Altman plot of the difference between the CockcroftGault (C-G)-estimated creatinine clearance and the iohexol GFR. The dashed line represents the mean difference between the respective MDRD or Cockcroft-Gault results and the iohexol GFR. Dotted lines represent the upper and lower limits of agreement (95\% CI).

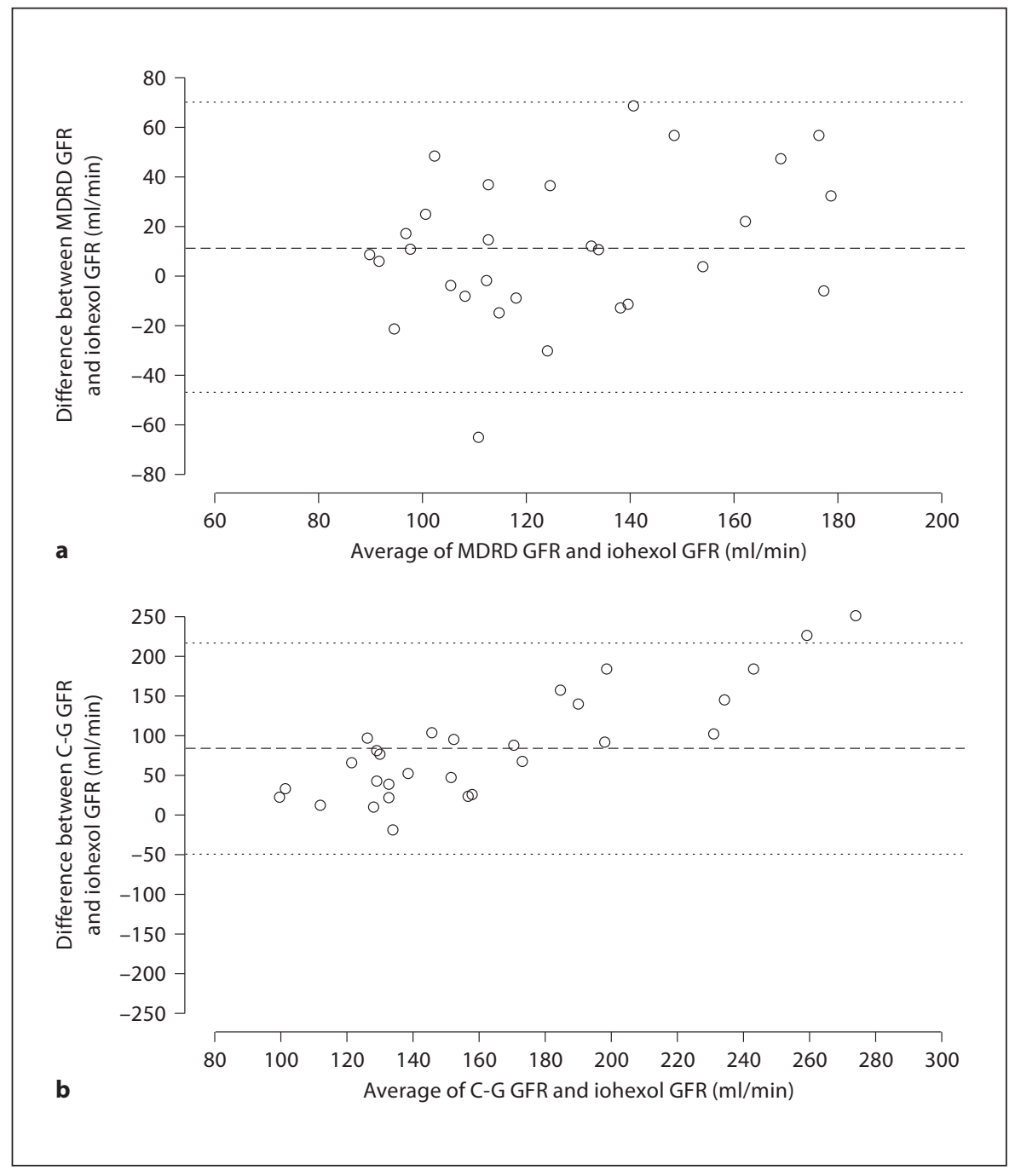

Table 4. Comparison of sampling point design

\begin{tabular}{|c|c|c|c|c|c|c|c|}
\hline \multirow[t]{2}{*}{ Parameter } & \multirow[t]{2}{*}{ Value } & \multicolumn{2}{|c|}{$\begin{array}{l}\text { Reference sampling design } \\
\text { (10 sampling points) }\end{array}$} & \multicolumn{2}{|c|}{$\begin{array}{l}\text { Optimal sampling design } \\
\text { (5 sampling points) }\end{array}$} & \multicolumn{2}{|c|}{$\begin{array}{l}\text { Brochner-Mortensen approach } \\
\text { ( } 5 \text { sampling points) }\end{array}$} \\
\hline & & SE & precision $(\%)^{1}$ & SE & precision $(\%)^{1}$ & SE & precision $(\%)^{1}$ \\
\hline $\mathrm{CL}, \mathrm{l} / \mathrm{h}$ & 7.06 & 0.68 & 9 & 0.88 & 12.4 & 0.93 & $13.1 \%$ \\
\hline $\mathrm{V}_{1}, \mathrm{l}$ & 9.87 & 3.12 & 32 & 3.74 & 37.9 & 4.12 & $41.7 \%$ \\
\hline $\mathrm{Q}, \mathrm{l} / \mathrm{h}$ & 11.4 & 3.1 & 27 & 3.72 & 32.6 & NA & NA \\
\hline $\mathrm{V}_{2}, \mathrm{l}$ & 6.15 & 0.88 & 14 & 1.144 & 18.5 & NA & NA \\
\hline
\end{tabular}

$\mathrm{CL}=$ Drug clearance (for iohexol, renal clearance assumed); $\mathrm{V}_{1}=$ volume of the central compartment; $\mathrm{Q}=$ flow between peripheral and central compartment; $\mathrm{V}_{2}=$ volume of the peripheral compartment; $\mathrm{SE}=$ standard error.

${ }^{1}$ Precision $=$ SE/value $(\%)$. 
Fig. 6. a Relationship between fat mass and iohexol GFR. b Relationship between lean body mass and iohexol GFR.
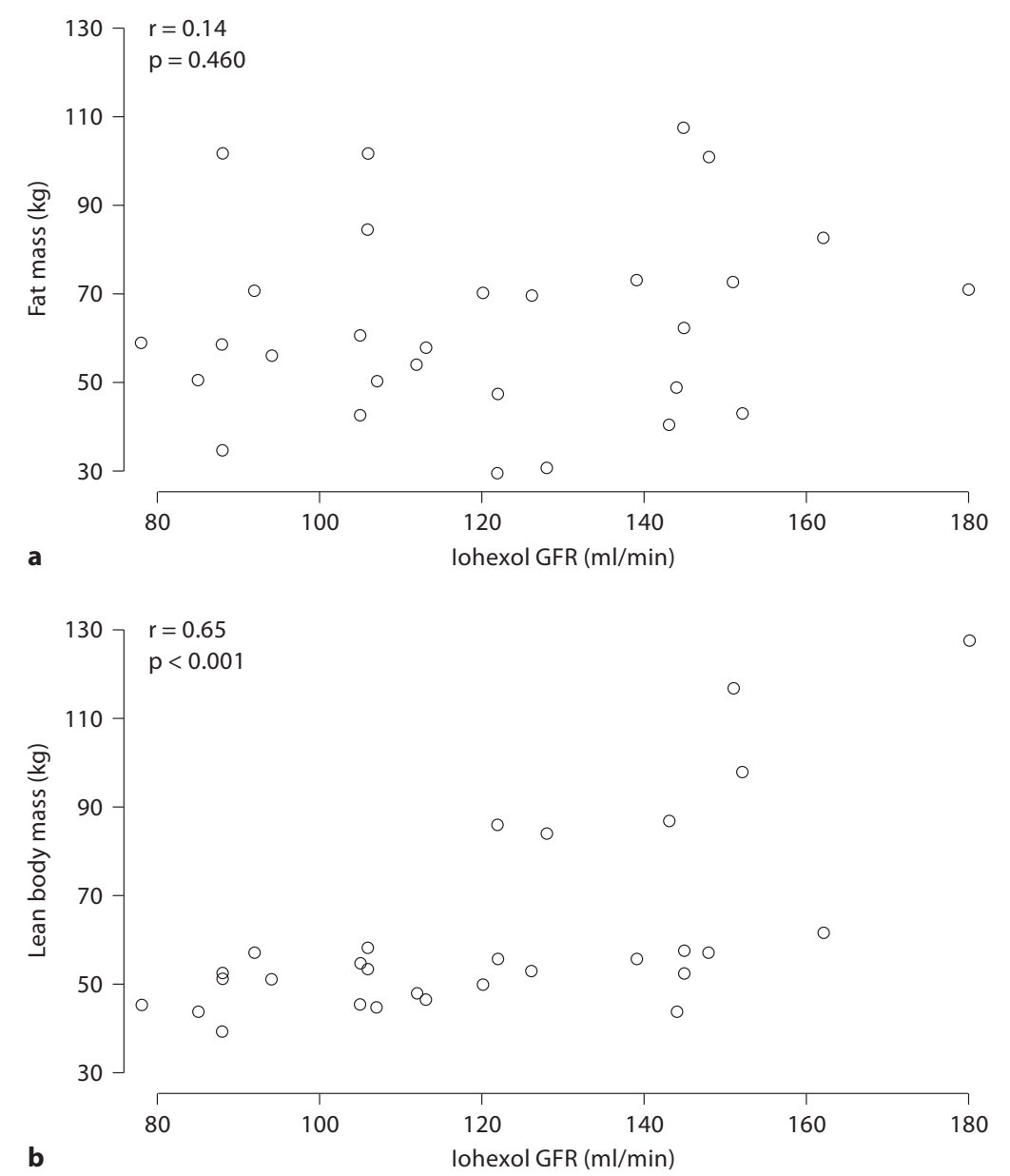

height $(\mathrm{r}=0.35, \mathrm{p}=0.067)$ or fat mass $(\mathrm{r}=0.14, \mathrm{p}=0.46)$ (fig. 6). Serum creatinine was inversely related to fat mass $(\mathrm{r}=-0.54, \mathrm{p}<0.002)$ and positively related to height $(\mathrm{r}=$ $0.43, \mathrm{p}=0.021)$, while no association was noted with lean body mass $(\mathrm{r}=-0.34, \mathrm{p}=0.076)$ or total weight $(\mathrm{r}=-0.14$, $\mathrm{p}=0.457)$. In contrast, cystatin $\mathrm{C}$ was not significantly associated with any anthropometric or body composition variables.

\section{Discussion}

Recent years have noted a convergence between the growing obesity problem and the emerging recognition of the existence of obesity-related kidney disease $[2,3,25$, 26]. These challenges require a reassessment of tradition- al GFR measurements and estimation methods, which were generally developed in lean populations with overt CKD $[15,27]$. To this end, we decided to study very obese individuals with apparently normal kidney function, since it is in this at-risk subgroup that incipient CKD will need to be identified and treated early. In doing so, we used population pharmacokinetics, a fairly novel method that has revolutionized the field of drug research and development [28].

Population pharmacokinetics offered to us a number of advantages over traditional pharmacokinetic methods, including the ability to (1) utilize data from all study visits, including those where sparse sampling for GFR measurement was performed (in this case, the 26 visits with 2 sampling points), (2) identify sources of variability within and between subjects (weight and lean body mass 
identified here as covariates) and (3) establish the simplest and most accurate iohexol sampling schedule in a particular population that could then be applied to clinical and research settings [29-31]. Only a handful of studies have thus far employed population pharmacokinetics to study GFR [32-35], with this being the first in obese individuals.

While iohexol is, for a variety of previously described reasons [36], an excellent GFR marker, it has never been studied specifically in obese adults, whose physiology and body composition may affect iohexol handling. We found that iohexol pharmacokinetics in obesity follows a two-compartment model, which confirms the existence of a 'peripheral' compartment into which iohexol is distributed (in addition to being excreted by the kidney). While this may not necessarily be surprising, it is a useful fact to establish for modeling purposes. In addition, the theoretical total volume of distribution, which reflects the relationship between serum iohexol concentrations and total body iohexol, was very similar to that previously observed in a group of healthy young men [37], suggesting that iohexol does not significantly distribute into the fat compartment.

The identification of an iohexol-based 5-point sampling schedule with windows of time during which the sampling should occur offers a convenient, flexible, and accurate method with which to measure GFR in obese subjects without overt CKD. We expect to use it in future studies involving similar populations. Schwartz et al. [36] derived a similar 4-point sampling schedule in a pediatric CKD population, though theirs was derived empirically rather than through pharmacokinetic modeling. Their sampling times included at least 2 points each on the early (fast) and later elimination curves (slow), as did ours, allowing for more accurate measurement of each of the individual slopes.

The characteristics of our obese cohort were similar to what others have found. While glomerular hyperfiltration was common, micro- or macroalbuminuria was noted in only a minority of patients [38-41]. That total weight and lean body mass (and not fat mass) were the 2 factors influencing GFR modeling supports the premise that the metabolic load is an important influence on GFR. This issue becomes relevant when addressing the ongoing controversy over indexing GFR by BSA or other physiological parameters [16]. Indexing by BSA is performed in order to fairly compare renal hemodynamics between people of varying body sizes. In the setting of obesity, however, body surface area is disproportionately affected by fat mass, which in our cohort did not significantly af- fect GFR. Therefore, indexing by BSA in obese individuals artificially lowers the GFR and masks any ongoing glomerular hyperfiltration. This is why we focused primarily on nonindexed measurements.

We found the endogenous filtration markers creatinine and cystatin C to be poorly correlated with GFR throughout the narrow ranges we studied. Though an ongoing controversy exists as to whether cystatin C is influenced by body composition [42-45], the lack of association between the two in this study suggests that it may in fact be a superior filtration marker under certain circumstances because it is less prone to being influenced by body composition. Correlations between GFR and estimating equations were superior to individual filtration markers though the Bland-Altman plots revealed at best modest agreement, suggesting that improved methods of GFR estimation are needed in this population. Of note, we found the MDRD equation overestimated GFR, which conflicts with previous observations $[8,46]$. This could be related to differences in the creatinine ranges studied, the prevalence of hyperfiltration, and/or the creatinine assays used.

Our study has a number of limitations. The cohort included mostly women with serum creatinine levels within a narrow range and was of modest size. This may have limited our statistical power and ability to describe important relationships. Whether our findings are applicable to men remains to be seen, though sex differences in iohexol pharmacokinetics have not been reported $[6,47]$. Having studied only obese individuals, we cannot comment on whether the optimal sampling time points would have been different for lean individuals. A recent report found that GFR measured over longer durations (i.e. up to $10 \mathrm{~h}$ ) may improve precision and accuracy [48]. However, that report included individuals with advanced kidney disease (i.e. estimated GFR of $32 \mathrm{ml} /$ $\mathrm{min} / 1.73 \mathrm{~m}^{2}$ and true median GFR of $48 \mathrm{ml} / \mathrm{min}$ ). That study population would be expected to have much slower plasma clearance and therefore require measurements over longer periods than the one profiled in this report. Therefore, our results may not necessarily apply to obese subjects with grossly abnormal serum creatinine levels. Finally, we chose not to measure iohexol urinary clearance as a comparison method because of the well documented difficulties in ensuring urinary collections are complete [36].

In the coming years, the ability to accurately measure GFR in obese individuals without overt CKD is likely to be increasingly important in both the clinical and research arenas. Furthermore, given the limited reliability 
of current GFR-estimating strategies in obese subjects that we and others have described, efforts should be made to identify more accurate methods of estimation. Our study offers a simple and accurate iohexol-based protocol with which to initiate such endeavors.

\section{Acknowledgements}

We greatly appreciate the assistance of Qian Li, MD, Mary Chambers, RN, and the Indiana Clinical and Translational Sciences Institute, Indiana Clinical Research Center, UL RR025761. Dr. Friedman (A.N.F.) is supported by the National Institute of Health (K23 RR019615). Dr. Lang Li (L.L.) and Dr. Stephen D. Hall's (S.D.H.) research is supported by NIH grant R01 GM74217.

\section{References}

1 World Health Organization: Obesity and Overweight Fact Sheet. http://www.who.int/ mediacentre/factsheets/fs311/en/ (accessed April 30, 2010).

-2 Hsu CY, McCulloch CE, Iribarren C, Darbinian J, Go AS: Body mass index and risk for end-stage renal disease. Ann Intern Med 2006;144:21-28.

- 3 Henegar JR, Bigler SA, Henegar LK, Tyagi SC, Hall JE: Functional and structural changes in the kidney in the early stages of obesity. J Am Soc Nephrol 2001;12:12111217.

4 Smith H: Diseases of the kidney and urinary tract; in Smith H (ed): The Kidney: Structure and Function in Health and Disease. New York, Oxford University Press, 1951, pp 836887.

-5 Brandstrom E, Grzegorczyk A, Jacobsson L, Friberg P, Lindahl A, Aurell M: GFR measurement with iohexol and ${ }^{51} \mathrm{Cr}$-EDTA. A comparison of the two favoured GFR markers in Europe. Nephrol Dial Transplant 1998; 13:1176-1182.

6 Gaspari F, Perico N, Ruggenenti P, et al: Plasma clearance of nonradioactive iohexol as a measure of glomerular filtration rate. J Am Soc Nephrol 1995;6:257-263.

7 Gaspari F, Perico N, Remuzzi G: Application of newer clearance techniques for the determination of glomerular filtration rate. Curr Opin Nephrol Hypertens 1998;7:675-680.

-8 Verhave JC, Fesler P, Ribstein J, du Cailar G, Mimran A: Estimation of renal function in subjects with normal serum creatinine levels: influence of age and body mass index. Am J Kidney Dis 2005;46:233-241.

-9 DuBois D, DuBois E: A formula to estimate the approximate surface area if height and weight be known. Arch Intern Med 1916;17: 863-871.

10 Das SK, Roberts SB, Kehayias JJ, et al: Body composition assessment in extreme obesity and after massive weight loss induced by gastric bypass surgery. Am J Physiol Endocrinol Metab 2003;284:E1080-E1088.

11 Diagnosis and classification of diabetes mellitus. Diabetes Care 2007;30(suppl 1):S42S47.

12 Chobanian AV, Bakris GL, Black HR, et al: Seventh report of the Joint National Committee on Prevention, Detection, Evaluation, and Treatment of High Blood Pressure. Hypertension 2003;42:1206-1252.
13 Shihabi ZK, Hinsdale ME: Serum iohexol analysis by micellar electrokinetic capillary chromatography. Electrophoresis 2006;27: 2458-2463.

14 Levey AS, Coresh J, Greene T, et al: Using standardized serum creatinine values in the modification of diet in renal disease study equation for estimating glomerular filtration rate. Ann Intern Med 2006;145:247-254.

15 Cockcroft D, Gault M: Prediction of creatinine clearance from serum creatinine. Nephron 1976;16:31-41.

16 Delanaye P, Radermecker RP, Rorive M, Depas G, Krzesinski JM: Indexing glomerular filtration rate for body surface area in obese patients is misleading: concept and example. Nephrol Dial Transplant 2005;20:20242028.

17 Team RDC: R: A Language and Environment for Statistical Computing. Vienna, R Foundation for Statistical Computing, 2008.

18 Jonsson E, Karlsson M: Xpose. http://www. xpose.sourceforge.net (accessed 2008)

19 Duffell S, Eccleston J, Kimko H, Denman N: WinPOPT User Guide. Dunedin, University of Otego, 2006.

20 Gamer M, Lemon J, Fellows I: irr: Various Coefficients of Interrater Reliability and Agreement. R Package Version 0.70, 2007.

21 Bland JM, Altman DG: Statistical methods for assessing agreement between two methods of clinical measurement. Lancet 1986;1: 307-310.

22 Beal B, Sheiner L: NONMEM User's Guide, Part I. San Francisco, University of California at San Francisco, 1992.

23 Sheiner LB, Rosenberg B, Marathe VV: Estimation of population characteristics of pharmacokinetic parameters from routine clinical data. J Pharmacokinet Biopharm 1977;5:445-479

24 James WP: WHO recognition of the global obesity epidemic. Int J Obes (Lond) 2008; 32(suppl 7):S120-S126.

25 Ejerblad E, Fored CM, Lindblad P, Fryzek J, McLaughlin JK, Nyren O: Obesity and risk for chronic renal failure. J Am Soc Nephrol 2006;17:1695-1702.

26 Kincaid-Smith P: Hypothesis: obesity and the insulin resistance syndrome play a major role in end-stage renal failure attributed to hypertension and labelled 'hypertensive nephrosclerosis'. J Hypertens 2004;22:10511055 .
27 Levey AS, Bosch JP, Lewis JB, Greene T, Rogers N, Roth D: A more accurate method to estimate glomerular filtration rate from serum creatinine: a new prediction equation. Modification of Diet in Renal Disease Study Group. Ann Intern Med 1999;130:461-470.

28 Bonate PL: Recommended reading in population pharmacokinetic pharmacodynamics. AAPS J 2005;7:E363-E373.

29 Ette EI, Williams PJ: Population pharmacokinetics II: estimation methods. Ann Pharmacother 2004;38:1907-1915.

30 Ette EI, Williams PJ: Population pharmacokinetics I: background, concepts, and models. Ann Pharmacother 2004;38:1702-1706.

- 31 Ette EI, Williams PJ, Lane JR: Population pharmacokinetics III: design, analysis, and application of population pharmacokinetic studies. Ann Pharmacother 2004;38:21362144.

32 Cole M, Price L, Parry A, et al: Estimation of glomerular filtration rate in paediatric cancer patients using ${ }^{51} \mathrm{Cr}$-EDTA population pharmacokinetics. Br J Cancer 2004;90:6064.

- 33 van Rossum LK, Mathot RA, Cransberg K, Vulto AG: Optimal sampling strategies to assess inulin clearance in children by the inulin single-injection method. Clin Chem 2003;49:1170-1179.

34 Wright JG, Boddy AV, Highley M, Fenwick J, McGill A, Calvert AH: Estimation of glomerular filtration rate in cancer patients. $\mathrm{Br}$ J Cancer 2001;84:452-459.

35 Martin L, Chatelut E, Boneu A, et al: Improvement of the Cockcroft-Gault equation for predicting glomerular filtration in cancer patients. Bull Cancer 1998;85:631-636.

-36 Schwartz GJ, Furth S, Cole SR, Warady B, Munoz A: Glomerular filtration rate via plasma iohexol disappearance: pilot study for chronic kidney disease in children. Kidney Int 2006;69:2070-2077.

37 Olsson B, Aulie A, Sveen K, Andrew E: Human pharmacokinetics of iohexol. A new nonionic contrast medium. Invest Radiol 1983; 18:177-182.

38 Ferris M, Hogan SL, Chin H, et al: Obesity, albuminuria, and urinalysis findings in US young adults from the Add Health Wave III study. Clin J Am Soc Nephrol 2007;2:12071214. 
-39 Navarro-Diaz M, Serra A, Romero R, et al: Effect of drastic weight loss after bariatric surgery on renal parameters in extremely obese patients: long-term follow-up. J Am Soc Nephrol 2006;17:S213-S217.

-40 de Jong PE, Verhave JC, Pinto-Sietsma SJ, Hillege HL: Obesity and target organ damage: the kidney. Int J Obes Relat Metab Disord 2002;26(suppl 4):S21-S24.

$\checkmark 41$ Chagnac A, Weinstein T, Korzets A, Ramadan E, Hirsch J, Gafter U: Glomerular hemodynamics in severe obesity. Am J Physiol Renal Physiol 2000;278:F817-F822.
42 Sharma AP, Kathiravelu A, Nadarajah R, Yasin A, Filler G: Body mass does not have a clinically relevant effect on cystatin C eGFR in children. Nephrol Dial Transplant 2009; 24:470-474.

43 Baxmann AC, Ahmed MS, Marques NC, et al: Influence of muscle mass and physical activity on serum and urinary creatinine and serum cystatin C. Clin J Am Soc Nephrol 2008;3:348-354.

44 Macdonald J, Marcora S, Jibani M, et al: GFR estimation using cystatin $\mathrm{C}$ is not independent of body composition. Am J Kidney Dis 2006;48:712-719.

-45 Vinge E, Lindergard B, Nilsson-Ehle P, Grubb A: Relationships among serum cystatin $\mathrm{C}$, serum creatinine, lean tissue mass and glomerular filtration rate in healthy adults. Scand J Clin Lab Invest 1999;59:587592.
46 Rigalleau V, Lasseur C, Perlemoine C, et al: Cockcroft-Gault formula is biased by body weight in diabetic patients with renal impairment. Metabolism 2006;55:108-112.

47 Krutzen E, Back SE, Nilsson-Ehle I, NilssonEhle P: Plasma clearance of a new contrast agent, iohexol: a method for the assessment of glomerular filtration rate. J Lab Clin Med 1984;104:955-961.

48 Agarwal R, Bills JE, Yigazu PM, et al: Assessment of iothalamate plasma clearance: duration of study affects quality of GFR. Clin J Am Soc Nephrol 2009;4:77-85. 